\title{
CARACTERIZACIÓN PSICOSOCIAL DEL CONSUMO DE MEDICAMENTOS EN COSTA RICA: ESTADO DE LA CUESTIÓN
}

\author{
PSYCHOSOCIAL CHARACTERIZATION OF MEDICATION \\ CONSUMPTION IN COSTA RICA: STATE OF THE ART
}

\author{
Massiel Arroyo Sibaja ${ }^{1}$ \\ massy2@gmail.com
}

Fecha de recepción: 20 noviembre 2013 - Fecha de aceptación: 26 mayo 2014

\begin{abstract}
Resumen
Se aborda el fenómeno del consumo de medicamentos mediante una revisión de la bibliografía disponible en el Sistema de Bibliotecas y Documentación de la Universidad de Costa Rica (SIBDI). No se encuentran investigaciones que aborden el concepto de medicamento desde la óptica de las personas consumidoras; por lo que se brinda una definición de medicamento basada en textos académicos y legales. Asimismo, se presenta brevemente la tendencia mayormente utilizada a nivel internacional en la investigación del consumo, uso, sub-utilización y adherencia al tratamiento medicamentoso, es decir la Atención Farmacéutica. De la misma se derivan modelos como el seguimiento farmacoterapéutico, la metodología Dáder, el modelo de Hepler y Strand, los cuales se exponen en el texto. Una limitación encontrada entre esas teorías, es que no ahondan sobre variables psicosociales y demográficas que afectan el consumo. En Costa Rica se encuentran cuatro investigaciones especializadas que intentan explicar el consumo de medicamentos desde el seguimiento farmacoterapéutico, y en algunos casos se incluyen variables de tipo demográficas como sexo, edad, estado civil, entre otras. Dentro de los resultados más relevantes se identifica una asociación entre edad, estado civil, nivel educativo con la utilización, la tenencia, consumo y disposición final de los medicamentos. También se encuentra que la metodología Dáder ha tenido éxito identificando y subsanando problemas relacionados con medicamentos. Sin embargo, no existe una propuesta que integre los múltiples factores relacionados al medicamento, al sistema de salud y variables psicosociales que caracterizan el consumo de medicamentos, de parte de ningún estudio consultado.

Palabras Clave: medicamento, medicina, salud, consumo de medicamentos, Seguimiento Farmacoterapéutico, farmacoterapia, Método Dáder, problemas relacionados con medicamentos, adherencia al tratamiento, Atención farmacéutica.
\end{abstract}

\begin{abstract}
It is address the phenomenon of medication consumption through a review of the literature available on the University of Costa Rica Library and Documentation System (SIBDI). There isn't found any research about the definition of medication from the people consuming this products; hence a definition based on legal and academic texts is given. Additionally it is presented the mainstream international tendencies to investigate consumption, use, underuse and treatment adherence, that is to say Pharmaceutical Care. From this theory derives different models like Drug therapy follow up, Dáder method, Hepler and Strand model, these are exhibit in the article. A limitation found in these theories is that they don't delve about psychosocial and demographic variables which affect the phenomenon of medication consumption. In Costa Rica were found four specialized investigations that intended to explain medication consumption using drug therapy follow up, and some of these studies also included psychosocial and demographic variables, such as sex, age, marital status. The results from these studies show an association between
\end{abstract}

1. Asistente, Escuela de Psicología, Sede Rodrigo Facio, Universidad de Costa Rica. 
age, marital status, and educational level with medication use, possession, consumption and disposal. There is also proven that Dáder method is a successful method to identify and correct pharmacotherapy failures. Nevertheless, there is not found an integrating model from any of the studies, comprehending variables related to medication, health systems and psychosocial and demographic characterization of medication consumption.

Keywords: medication, medicine, health, medication consumption, drug therapy follow up, pharmacotherapy, Dáder Program, pharmacotherapy failures, Pharmaceutical Care, Pharmaceutical Treatment.

\section{Introducción}

El consumo de medicamentos es una de las principales prácticas que las personas realizan ya sea para mantener la salud o para recuperarla. El sistema de salud costarricense se caracteriza por una fuerte tendencia al uso de fármacos en la atención de la salud. Durante el 2011, la Caja Costarricense de Seguro Social despachó 71 millones de medicamentos, lo cual representó un gasto de $\square 100.000$ millones de colones al sistema de salud (Caja Costarricense de Seguro Social, 2012). Sin embargo, el entendimiento de lo que es un medicamento, el para qué y cómo se usa es difuso entre las personas consumidoras. Al ser el medicamento utilizado con tanta frecuencia desde variedad de ámbitos, el esfuerzo investigativo por definir este concepto debe tomar en cuenta las distintas connotaciones y usos que se le pueden atribuir al medicamento. Del mismo modo, debe contemplar las prácticas que se enmarcan en una serie de características psicosociales que las personas consumidoras de medicamentos poseen.

La poca claridad que poseen las personas consumidoras sobre la definición de este concepto puede conducir a consecuencias negativas, que afectarán el uso que se le dé al mismo. Dentro de los ejemplos más frecuentes está el incumplimiento del tratamiento farmacológico, mal uso, desperdicio del medicamento y agravamiento de la enfermedad como consecuencias de una falta de comprensión por parte de la persona consumidora. Este tema ha sido de interés para profesionales de la salud que se han percatado de la problemática de la subutilización de los medicamentos, por lo tanto, se han realizado investigaciones en torno al uso, sub-uso y adherencia al tratamiento médico en Costa Rica, las cuales han presentado información que ayuda a explicar este fenómeno. Algunas de estas investigaciones utilizan metodologías internacionales avaladas y recomendadas para el estudio de esta temática, mientras que otras incluyen descripciones de las asociaciones entre variables psicosociales y demográficas con el consumo de medicamentos.

Se intentará en este artículo una aproximación teórica al término medicamento, definiendo el mismo a partir de material bibliográfico académico y no académico. Igualmente se considerarán otros conceptos asociados al mismo, tales como seguimiento farmacéutico y problemas relacionados con medicamentos. Por último, se explorarán investigaciones realizadas en Costa Rica en torno al concepto de medicamento, su utilización, subutilización y adherencia al tratamiento médico. Sin embargo, se decide no incluir las investigaciones realizadas sobre adherencia a tratamientos exclusivos para enfermedades específicas; ya que estos abordan la problemática del consumo y la adherencia desde grupos poblacionales selectos que presentan una necesidad de consumo específica. Es decir estas investigaciones tienen un alto grado de especificidad en sus objetivos y restringen el problema según enfermedad y grupo de medicamentos; este artículo se centra en el consumo de medicamentos en población general. Para consulta sobre algunos de estos estudios se puede referir a las investigaciones de Güell (2002) sobre adherencia al tratamiento retroviral; Troncoso Pantoja, Delgado Segura y Rubilar Villalobos (2013) sobre adherencia al tratamiento en pacientes con Diabetes tipo 2; Jiménez y Fernández (2008) presentan una caracterización del consumo de medicamentos en población adulta mayor; Mesén Arce y Chamizo García (2005) estudiaron los determinantes del ambiente familiar y la adherencia al tratamiento 
de la tuberculosis. Estos son solo una muestra de los estudios que se pueden encontrar de este tipo en Costa Rica.

Igualmente se excluyen los resultados de la Encuesta Nacional de Salud (ENSA 2006) (Hernández y Sáenz, 2006) y la Encuesta Nacional sobre consumo de drogas (López, 2012). En estas encuestas se abordó el consumo de medicamentos desde la prevalencia del uso de grupos medicamentosos en el caso de ENSA 2006, asociaciones con enfermedades, felicidad; se abordó igualmente el tema de la automedicación, uso y disposición final de los medicamentos (Jiménez-Herrera, 2008). En el caso de la Encuesta Nacional sobre drogas, esta se orienta al abuso de medicamentos con prescripción médica (opiáceos, estimulantes y tranquilizantes). Por lo tanto no se aborda la problemática desde las características psicosociales o bien del medicamento que afecta el consumo en población general.

Para esta investigación se realizó una búsqueda bibliográfica para definir medicamento desde una perspectiva médica y farmacéutica; además de incluir el estado de la cuestión sobre el consumo de medicamentos en Costa Rica, especializado a la investigación que se ha encargado de encontrar factores sociodemográficos que incidan en la práctica de consumo. La búsqueda se realizó en las bases de datos del Sistema de Bibliotecas Documentación e Información (SIBDI) de la Universidad de Costa Rica. Para definir palabras claves utilizadas en la búsqueda se llevaron a cabo reuniones con expertos sobre el tema, quienes orientaron la misma. Además se consultaron diccionarios y glosarios disponibles en la colección general de las bibliotecas anteriormente mencionadas.

\section{Acerca del concepto de medicamento}

Cuando se piensa en medicamentos las imágenes que llegan a la mente pueden ser muy diversas, algunas personas pueden pensar en cápsulas, otras en inyecciones y otras en plantas. Y si se intenta definir con palabras, las respuestas que se obtienen pueden ser incluso más diversas y variadas de lo que se piensa. No se puede partir del hecho de que todas las personas compartimos el concepto de medicamento. Esta falta de claridad lleva a que las personas juzguen algunas sustancias como medicamentos y otras no; afectando el uso que se le da a estas sustancias.

Tinoco (2007) indica que la oferta comercial-publicitaria de medicamentos altera en primer lugar, las percepciones que tienen las personas con respecto a los alcances que tiene el medicamento y en segundo lugar, la demanda de medicamentos; que a su vez interfiere en el uso que se realiza de los mismos. Los esfuerzos por sistematizar y estandarizar la práctica de la selección y prescripción de medicamentos por parte de agentes de salud son rigurosos y responden a reglamentos establecidos por el Ministerio de Salud, entre otras instituciones involucradas. No obstante, aún existe una alta subutilización de medicamentos entre la población general (Jiménez-Herrera, 2008; Jiménez-Herrera y Fernández-Rojas, 2008; Jiménez, 2004, 2006), lo cual indica que la población general consumidora de los mismos no tiene la misma claridad que se maneja desde el sector salud, en cuanto a utilización, dosificación, prescripción, manejo y desecho (Caja Costarricense de Seguro Social, 2012; Jiménez, 2004, 2006). Se conoce a partir de diferentes investigaciones realizadas por la CCSS que el $15 \%$ de las personas no cumple con el tratamiento médico prescrito, más del $68 \%$ de las personas botan al basurero la medicación sobrante, el $62 \%$ de las personas salen insatisfecho(a)s del consultorio cuando no les dan una receta, el $23 \%$ comparte los medicamentos que se recetan por el sistema de salud, y no se retiran de las farmacias 72.000 recetas al mes (Caja Costarricense de Seguro Social, 2012).

Los datos anteriores demuestran que no se comparten buenas prácticas de utilización de medicamentos en la población general. Las definiciones, concepciones y prácticas que se manejan desde el sector salud no calan en la población de la forma esperada, a pesar de los esfuerzos por educar a la población. Por este motivo se ha intentado investigar los factores psicosociales y demográficos que intervienen en el consumo de medicamentos e identificar desde esta área si existen problemas asociados a las características psicosociales de las personas que puedan afectar el consumo de medicamentos. Sin embargo, en los textos consultados sobre esta temática no se 
encuentra una definición de medicamento única y exclusiva, se encuentran por tanto definiciones similares. De ahí que para este artículo sea necesario definir qué es un medicamento para el sector salud, antes de presentar el estado de la cuestión sobre la caracterización psicosocial del consumo de medicamentos.

El término medicamento está definido en diccionarios como "Agente o sustancia simple o compuesto, que se administra al exterior o al interior con objeto terapéutico. Fármaco u otra sustancia utilizada como medicina" (Capdevilla, 1981; Villanueva, 2000; Zamora, 2005). Arias le asigna dos acepciones al medicamento, una se refiere al principio activo o fármaco; mientras que la otra hace referencia al medicamento como "producto farmacéutico empleado para la prevención, diagnóstico o tratamiento de una enfermedad o estado patológico o para modificar sistemas fisiológicos en beneficio de la persona a quien le fue administrado" (1999, p. 141). Se realiza la diferenciación entre medicamento como principio activo o fármaco; y el medicamento en su presentación como tratamiento. Así, se acepta una u otra acepción respecto al uso que se destine al medicamento, por ejemplo, sería un principio activo en caso de que se haga investigación con esa sustancia, pero si es para administrarse con el fin de curar una enfermedad, entraría en la segunda definición, como tratamiento.

De la mano con las definiciones aportadas anteriormente se encontró la definición oficial o legal de medicamento. En la Ley General de Salud (Ley 5395), se define medicamento como "toda sustancia o productos naturales, sintéticos o semi-sintéticos y toda mezcla de esas sustancias o productos que se utilicen para el diagnóstico, prevención, tratamiento y alivio de las enfermedades o estados físicos anormales, o de los síntomas de los mismos y para el restablecimiento o modificación de funciones orgánicas en las personas o en los animales" (Ministerio de Salud, 1973, Art. 104). Partiendo de esta definición se reconoce que los medicamentos abarcan una amplia gama de sustancias o prácticas que tienen como fin último preservar, diagnosticar y mejorar la salud, tanto en animales como humanos. Se excluye de esta definición de medicamento, los principios o sustancias activas utilizadas en análisis químicos y clínicos. Pero se incluyen sustancias como alimentos, cosméticos, entre otros, que hayan sido adicionadas con sustancias medicinales.

Por lo tanto, en la ley no se hace referencia a los medicamentos homeopáticos como tales, pero al tener estos una sustancia medicinal incorporada se contempla como medicamentos. Por lo tanto, no son medicamentos la medicina natural, como la fitoterapia. Tanto la medicina homeopática como la alopática pueden provocar problemas de salud si se administran de forma equivocada y sin supervisión médica o farmacéutica. Todo medicamento tiene efectos secundarios asociados y determinados previamente por los especialistas; pero además de los efectos secundarios pueden presentarse reacciones adversas imprevistas, las cuales dependen de la respuesta fisiológica de cada persona. A raíz de esto, se hace necesaria la supervisión médica y farmacéutica en el consumo de medicamentos.

Ahora bien, otro punto a considerar es la idea que tienen las personas usuarias de servicios de salud y consumidoras de medicamentos sobre lo que es medicamento o sus categorías. Como ya se mencionó, debe existir un alto nivel de acuerdo entre las definiciones disciplinares y las de las personas consumidoras para evitar riesgos a la salud, un mal aprovechamiento de los medicamentos y de los recursos económicos disponibles.

La falta de información investigativa sobre el tema dificulta determinar a ciencia cierta si existe algún nivel de acuerdo entre la perspectiva médica/farmacéutica y la perspectiva del usuario frente a lo que es un medicamento y sus posibles efectos positivos o negativos. De existir un nivel de acuerdo o coordinación entre el sistema de salud y personas consumidoras, dicho nivel de acuerdo incidirá en los resultados esperados que debe tener un medicamento; que de acuerdo a las fuentes consultadas son: a. Diagnosticar o curar la enfermedad, b. Reducir o eliminar los síntomas, c. Parar o ralentizar el proceso de una enfermedad y d. Prevenir la enfermedad o síntomas.

Adicionalmente a las dificultades nacidas de la discrepancia en la definición de medicamento que los usuarios y profesionales tienen, existen una serie de consecuencias de la particular estrategia conductual que cada persona defina para el consumo. Al administrarse un medicamento 
existen riesgos de que este provoque efectos secundarios o bien reacciones adversas derivadas de una inadecuada subutilización. Esto traerá consecuentemente resultados no óptimos, tales como complicaciones clínicas, progresión de la enfermedad, necesidad de atención especializada, etc. Por lo tanto, se hace necesaria más investigación acerca de los factores determinantes en el consumo, utilización, sub-utilización y adherencia al tratamiento farmacológico en población general. Esto incluiría la investigación tanto de tratamientos medicamentosos para enfermedades específicas y el consumo de medicamentos (con y sin prescripción médica) entre la población general.

\section{Modelos de evaluación y atención al consumo inadecuado de medicamentos}

En la literatura sobre el uso de medicamentos se encuentran varios modelos explicativos de los factores involucrados en el utilización/subutilización y adherencia al tratamiento farmacológico. Estos modelos se circunscriben dentro de la práctica de la Atención Farmacéutica (Pharmaceutical Care, su nombre en inglés), es una disciplina reconocida a nivel mundial y apoyada por la Organización Mundial de la Salud y la Organización Panamericana de la
Salud. La OMS en el Informe de Tokio (1993) reconoce la labor de Atención Farmacéutica y la define como:

“...un compendio de práctica profesional, en el que el paciente es el principal beneficiario de las acciones del farmacéutico y reconoce que esta Atención Farmacéutica es el compendio de las actitudes, los comportamientos, los conocimientos, las responsabilidades y las destrezas del farmacéutico en la prestación de la farmacoterapia, con objeto de lograr resultados terapéuticos definidos en la salud y la calidad de vida de cada paciente.” (p.3)

Basándose en el papel que debe tener la disciplina farmacéutica en el sistema de salud y tomando en cuenta las directrices de la Atención Farmacéutica se desarrollan teorías, modelos y protocolos de intervención. Se incluyen en estos modelos características del sistema de salud, de la atención brindada por profesionales, características del medicamento, idiosincrasia y particularidades de los y las usuarias. Dentro de estos modelos se encuentra el de Hepler y Strand propuesto en 1990 (American Society of Hospital Pharmacists, 1993; Hepler y Strand, 1990; Quesada y Jaén, 2004). Este modelo abarca la acción del médico(a), la distribución y las acciones del paciente; y se desglosa de la siguiente manera:

Figura 1

Modelo de atención a problemas relacionados con medicamentos de Hepler y Strand

\begin{tabular}{|c|l|}
\hline Prescripción inapropiada & $\begin{array}{l}\text { - Régimen inapropiado } \\
\text { - Régimen innecesario }\end{array}$ \\
\hline Distribución inapropiada & $\begin{array}{l}\text { - El medicamento no está disponible cuando se necesita } \\
\text { - Error de dispensación }\end{array}$ \\
\hline $\begin{array}{c}\text { Comportamiento } \\
\text { inapropiado del paciente }\end{array}$ & $\begin{array}{l}\text { - Cumplimiento de un régimen inadecuado } \\
\text { - Incumplimiento de un régimen adecuado }\end{array}$ \\
\hline Idiosincrasia del paciente & $\begin{array}{l}\text { - Respuesta idiosincrática al medicamento } \\
\text { - Error o accidente }\end{array}$ \\
\hline Monitorización & $\begin{array}{l}\text { - Fallo en detectar y resolver una decisión terapéutica } \\
\text { inapropiada } \\
\text { inapropiada }\end{array}$ \\
\hline
\end{tabular}

Elaboración propia basado en Hepler y Strand (1990) y Quesada y Jaén (2004). 
Los factores presentados inciden básicamente en el consumo, discontinuación del tratamiento medicamentoso y en la adherencia al tratamiento médico, pero no detalla sobre aspectos idiosincráticos de los y las consumidoras. Por su parte, los factores concernientes al seguimiento que se brinda por parte del personal de salud se han manejado de múltiples maneras con gran éxito, como se expondrá más adelante, el uso de metodologías para prevenir la subutilización de medicamentos ha tenido buen resultado a nivel internacional y nacional. Sin embargo, los factores concernientes al comportamiento e idiosincrasia de las personas usuarias no están extensamente estudiados o solventados; y es poco lo que se conoce acerca de características psicosociales que intervienen en el consumo y en la adherencia.

Cuando se identifica un fallo en alguno de los factores del modelo anterior se suele identificar problemas causados por medicamentos (PRM). Los PRM son aquellos que se derivan del uso de la farmacoterapia, los cuales tienen varias causas y conducen a la no consecución del objetivo terapéutico o a la aparición de efectos no deseados debidos principalmente a la terapia farmacológica (Grupo de investigación en Atención Farmacéutica de la Universidad de Granada, 2007). Se concuerda que existe un problema relacionado con medicamentos cuando se percibe alguna anormalidad en el proceso terapéutico que afecte negativamente al usuario y la usuaria. Dentro de los posibles PRMs se encuentran problemas al no contar con medicación necesaria, poca o baja efectividad del medicamento, entre otros. El Consenso de Granada incluye como causas de los problemas relacionados con medicamentos aquellas dependientes del medicamento, dependientes del y la paciente, dependientes de quien prescribe, del farmacéutico(a) y del sistema.

Por lo tanto, el consumo de medicamentos debe verse como un proceso dialéctico con múltiples factores que pueden alterar la buena consecución del mismo. Se ha investigado la línea de ejecución hasta llegar a la persona que consume, pero no se cuenta con suficiente información que aclare qué mecanismos juegan parte al final del proceso, es decir en el destinatario del medicamento.
Con respecto a los factores psicosociales que determinan los PRM, Jiménez (2004, 2006), propone que estos pueden ser demográficos, como sexo, edad, etc.; sociopsicológicos como la personalidad, clase social, educación, económicos; los estructurales que se refieren al conocimiento de la enfermedad y del medicamento y a las percepciones de las personas. Aunado a esto Jiménez (2004) también identifica otros factores que inciden en la población adulta mayor para el incumplimiento de los tratamientos farmacológicos; estos se dividen en dos categorías:

1) Los relacionados con el paciente y el médico, como por ejemplo el tipo de diagnóstico, bajo nivel educativo y socioeconómico del paciente, insatisfacción con la relación paciente/ médico y las relaciones familiares.

2) $Y$ factores relacionados con la terapia como la polifarmacia, es decir, la prescripción de varios medicamentos a la vez; dosificación de la medicación; costo de tratamiento; instrucciones claras; desinformación, entre otras.

Como ya se expuso el abordaje de los PRM relacionados con la prescripción y la terapia se han atendido a nivel nacional desde modelos internacionales, tal es el caso de la práctica del seguimiento farmacoterapéutico. El seguimiento farmacoterapéutico es una práctica del área de la planificación farmacéutica, que a su vez está basada en la Atención Primaria de Salud, propuesta de la OMS, para lograr que la atención en salud sea orientada a los usuarios logrando una mayor equidad en los servicios de salud (Organización Mundial de la Salud, 1993; Organización Panamericana de la Salud, 2011). Este consiste en brindar atención a las personas usuarias y está relacionado con "la detección, prevención y resolución de problemas relacionados con la medicación, de forma continuada, sistematizada y documentada, en colaboración con el propio paciente y con los demás profesionales del sistema de salud, con el fin de alcanzar resultados concretos que mejoren la calidad de vida del paciente" (Quesada y Jaén, 2004, p. 40).

Siguiendo la metodología de atención del seguimiento farmacoterapéutico se encuentra el Método Dáder; el cual fue creado por el Grupo 
de Investigación en Atención farmacéutica de la Universidad de Granada en el 2003. Consiste en el seguimiento y acompañamiento del tratamiento farmacológico del usuario mediante la "obtención de la Historia Farmacoterapéutica del paciente, esto es los problemas de salud que presenta y los medicamentos que utiliza, y la evaluación de su Estado de Situación a una fecha determinada, para identificar y resolver los posibles Problemas Relacionados con Medicamentos que el paciente pueda estar presentando" (Machuca-González, Faus-Dáder y Fernández-Llimós, 2003, p. 5).

El Método Dáder implica una relación cercana, a través del tiempo entre el farmaceuta y el paciente, con el fin de poder solucionar los PRM que surjan. Consta de tres fases: a. Obtención de información mediante cuestionarios; b. Desarrollo del plan de seguimiento y c. Evaluación del seguimiento. Asimismo, el Método Dáder cuenta con mucha popularidad en Costa Rica y se ha implementado tanto en farmacias de la Caja Costarricense de Seguro Social, así como en farmacias de comunidad utilizando muestras diversas.

Bajo la perspectiva de la investigación farmacéutica de los PRM se ha logrado intervenir en aquellos factores dependientes del sistema de salud, pero se ha dejado de lado la intervención con respecto a la idiosincrasia de la persona usuaria; por lo que la investigación en esta área podría indicar caminos de intervención a futuro, en especial desde las ciencias sociales. Conocer aspectos psicosociales de los y las consumidoras de medicamentos serviría para poder comprender qué piensan sobre el medicamento, determinar las prácticas en torno al consumo del mismo y poder esclarecer las motivaciones sociales, psíquicas, conductuales, culturales, económicas, etc. que llevan a las personas a actuar como lo hacen cuando deben apegarse a un tratamiento farmacológico, o simplemente tomar la decisión de consumir un medicamento o no.

Por lo tanto, la colaboración interdisciplinaria entre las ciencias sociales y las ciencias de la salud es requisito para integrar los conocimientos que se manejan actualmente y las necesidades de investigación futura; investigación que permita dilucidar el consumo de medicamentos en la población costarricense. Actualmente los estudios realizados abarcan aspectos sobre el tratamiento, caracterización psicosocial de comunidades o grupos, intervenciones frente a los PRM mediante la metodología Dáder y adherencia a tratamientos específicos para enfermedades.

\section{Investigaciones realizadas en Costa Rica}

Las investigaciones realizadas en torno al uso de medicamentos no bastan para ahondar en lo que significa tal concepto para las personas usuarias y, en menor medida, los factores que inciden en la decisión de consumo. Sin embargo, tales investigaciones han logrado abordar implícitamente el concepto de medicamento desde perspectivas sociales, económicas y comunitarias. Así pues, se han desarrollado en la Universidad de Costa Rica, por parte de las Escuelas de Farmacia y Medicina investigaciones sobre la adherencia al tratamiento médico (Jiménez, 2004; Rodríguez, Martínez y Villalobos, 1996); y estudios de seguimiento farmacoterapéutico (Carazo y Muñoz, 2004; Quesada y Jaén, 2004). Tales investigaciones abordan el problema del consumo del tratamiento farmacéutico desde la óptica del seguimiento farmacéutico y en menor medida, desde los factores sociales (conocido como idiosincrasia del paciente) que determinan el consumo y utilización de los medicamentos.

Por parte de la Escuela de Farmacia de la Universidad de Costa Rica, se encuentran los proyectos de Quesada y Jaén (2004) y Carazo y Muñoz (2004). Estos autores contribuyeron al tema aportando evidencia de la efectividad del seguimiento farmacéutico mediante la utilización de la metodología Dáder. En el caso de Quesada y Jaén (2004) se evaluó la metodología Dáder en el seguimiento farmacéutico de pacientes con Diabetes Mellitus. Se estudió una muestra de 40 personas (14 hombres, 26 mujeres), a quienes se les identificaron problemas relacionados con medicamentos. En una primera etapa se utilizaron cuestionarios para la obtención de la historia del usuario y usuaria y se procedió a identificar los PRM (24 personas presentaron alguno de estos problemas) y se evaluó si era posible la intervención, la cual se realizó con ayuda del farmaceutico, el paciente y el médico.

En primer lugar y en la mayoría de los casos los problemas relacionados con medicamentos 
identificados, correspondían a no recibir una medicación que necesitaban, en aproximadamente la mitad de los casos evaluados. El segundo PRM en incidencia fueron los relacionados a la prescripción de un tratamiento que no se necesita, en aproximadamente $33 \%$ de los casos. En tercer lugar se encontraron problemas referentes a la peligrosidad asociada al medicamento por una dosis alta o por incumplimiento de la terapia $(25 \%)$. Como se puede apreciar los dos primeros PRM dependen de la terapia farmacológica y no de la persona consumidora, sin embargo el tercer lugar corresponde a incumplimiento de terapia; esta variable está totalmente asociada a la persona consumidora, pero no se ahonda más en las causas que provocan el incumplimiento.

Además, se comparó la efectividad de la intervención con una medición previa a la aplicación de la metodología Dáder y otra medición al finalizar el programa, considerando como exitoso el programa si los PRM se reducían en cada caso. Los autores determinaron que el Método Dáder es una herramienta útil y eficaz para la identificación y solución de problemas relacionados con medicamentos, en especial en aquellos casos que involucran al medicamento y la relación prescriptor/paciente. Una limitación del estudio es que el mismo no abarca aspectos sociales o idiosincráticos de las personas participantes.

Por su parte, Carazo y Muñoz (2004) investigaron la eficacia de la aplicación del Método Dáder en un grupo de personas adultas mayores. La metodología consistió en el uso del mismo cuestionario anteriormente mencionado para indagar la historia del paciente y realizar el diagnóstico de los problemas relacionados con medicamentos. Utilizaron una muestra de 63 personas, encontraron un total de $32 \mathrm{PRM}$ en la muestra total. El 50\% de los PRM encontrados pertenecen a la categoría de necesidad, es decir necesitar un medicamento no disponible o no necesitar un medicamento prescrito. Aproximadamente 1 de cada 10 pertenece a la categoría de seguridad, por ejemplo un medicamento que podría ser inseguro dependiendo de la dosificación y el consumo. Por último, 4 de cada 10 PRM son identificados como problemas de efectividad del tratamiento. Por consiguiente, se investigaron las posibles causas de los problemas, encontrando que el incumplimiento del tratamiento es una de las causas principales (6.1\%), se evaluaron también la interacción y la duplicidad, pero en ninguna de estas se encontró un porcentaje que indicara causalidad. Sin embargo, en la categoría de otras causas (93.9\%) se descubrió en primer lugar que $48.5 \%$ del porcentaje de otros se debe a la falta del medicamento, el $24.2 \%$ a falta de respuesta terapéutica tanto del médico como del farmacéutico; en tercer lugar a las reacciones adversas (10.6\%), los problemas relacionados con medicamentos debido a la dosis (alta/baja) que representaron el $7.6 \%$, y por último los asociados a la prescripción de un medicamento no adecuado que presentó un 3\%. Del total de problemas identificados, los autores lograron resolver el $72.5 \%$ con la metodología Dáder. Se rescata de esta investigación que el incumplimiento del tratamiento medicamentoso es una causa directa de los PRM, pero no se brinda información que explique por qué se da el incumplimiento del tratamiento medicamentoso en esta muestra.

En cuanto a las investigaciones realizadas en torno a la identificación de factores psicosociales que inciden en la adherencia al tratamiento, se encontraron dos en el país. Por una parte, Rodríguez et al. (1996) determinaron la incidencia de factores psicosociales en el uso de medicamentos en el adulto mayor. La investigación se realizó con 60 adultos mayores en la comunidad de Cocorí de Cartago. Propusieron categorías psicosociales que incidieron en el uso de medicamentos: aspectos sociodemográficos, características de la familia, características del entorno y características del uso del medicamento. Encontraron que 6 de cada 10 personas en la muestra no ingirieron los medicamentos de la forma en que fueron recetados por el médico; se incluye principalmente la incapacidad de recordar aspectos relacionados con la ingesta o aplicación del medicamento (70\%), falta de apoyo familiar (4 de cada 10 personas), dependencia económica y relación médico-paciente (5 de cada 10 dijeron recibir poca información en el centro de salud).

Por otra parte, Jiménez (2004) analizó la subutilización, desecho y disposición final de los medicamentos en el área de salud de Coronado. Específicamente se buscó asociaciones entre factores demográficos como edad, género y 
escolaridad con la tenencia de medicamentos en el hogar, su uso incluyendo almacenamiento de medicamentos, incumplimiento de tratamiento y disposición final de los mismos. El estudio abarcó 440 hogares y se utilizó un instrumento tipo encuesta para determinar las prácticas asociadas a la utilización, subutilización y disposición final de los medicamentos. El cuestionario se subdividió en tres categorías: determinantes de la salud individual, uso de medicamentos y almacenamiento de medicamentos en el hogar.

El autor encontró que factores demográficos como edad, nivel educativo y sexo, entre otros, se asociaron significativamente a la cantidad de medicamentos encontrados en el hogar. Entre ellos los que más influyen son el estado civil y la escolaridad, por ejemplo personas solteras o divorciadas presentaron mayor tenencia de medicamentos en el hogar, al igual que personas con baja escolaridad.

Un factor que afecta la utilización del medicamento es el hecho de tener que recordar datos acerca del mismo, tales como cuál medicamento ingerir, las horas de ingesta para cada medicamento, si se ingiere con alimentos, etc.; en este caso un $25 \%$ de la muestra reportó tener problemas relacionados con medicamentos como consecuencia. El autor reporta que entre mayor conocimiento tenga la persona de los factores que pueden afectar al medicamento, se tiende significativamente a conservarlos más. Igualmente se tiende a conservar los medicamentos en los casos en los que la persona tiene mayor dificultad para manipular el medicamento y seguir instrucciones.

Jiménez (2004) también encontró que en los casos en los cuales el tratamiento se percibe ineficiente, el $29.8 \%$ de la muestra reportó no continuar con el tratamiento, mientras que el $23.9 \%$ consulta con el personal de salud. Otros factores que influyeron igualmente en la tenencia de medicamentos en el hogar fueron el uso de tratamientos alternativos o no alopáticos (por ejemplo la homeopatía y medicina natural), factores de nivel estructural como el conocimiento de la enfermedad y factores del uso y manejo del medicamento.

Con respecto a la subutilización, el estudio determinó que la falta de información y medicamentos vencidos son la principal causa de la subutilización. Del total de la muestra 50\% desconocían aspectos relevantes del medicamento, desconocían efectos secundarios un $42 \%$, advertencias y precauciones un $40 \%$, aspectos sobre el almacenamiento un $45 \%$ y sobre el desecho de los medicamentos un 55\% del total de participantes. Estos datos son alarmantes si se piensa desde los costos económicos y los costos para la salud de las personas. A partir de esta investigación se dilucidan algunos factores psicosociales relevantes como los ya mencionados. Además, se intenta describir aspectos que forman parte de la conducta de consumo, tales como conocimiento sobre la medicación; no obstante, no se asocian estos factores entre sí para establecer un modelo explicativo integrador sobre el consumo de medicamentos.

Las investigaciones que se encuentran en el país aportan posibles variables asociadas a la utilización y subutilización del medicamento, sin embargo no existe un panorama claro ni modelos que expliquen conductualmente el uso que se le da al medicamento. Como primer paso para un acercamiento comprensivo a estas prácticas se debe definir qué es un medicamento, desde la perspectiva académica, de salud y desde las mismas personas usuarias. En segundo lugar, se debe realizar un abordaje integrador, preferiblemente multidisciplinario del consumo de medicamentos. La información actual indica que existe una incidencia de factores psicosociales, económicos, familiares y relacionales con el personal de salud en la conducta de consumo de medicamentos. Pero, como se expuso, la investigación realizada hasta ahora no es suficiente para establecer asociaciones e interrelaciones entre todos esos factores.

\section{Conclusiones}

En la revisión de literatura realizada no se encontró para Costa Rica evidencia de que el concepto de medicamento esté definido de igual manera entre los sectores de salud y las personas usuarias del sistema de salud. Tampoco existe investigación que se centre en definir el concepto basándose en las opiniones y creencias de la población general. De hecho no hay evidencia de que el concepto de medicamento utilizado en disciplinas de la salud sea el mismo encontrado en 
los diccionarios o en la Ley General de Salud; ya que en ninguna de las investigaciones consultadas se encuentra una definición de medicamento. Esta carencia de delimitación, coherencia y definición implica diferentes maneras de vivir el proceso de la medicación, que en algunos casos puede resultar en problemas relacionados con medicamentos en detrimento de la salud del usuario.

Las investigaciones consultadas han utilizado el Método Dáder de seguimiento farmacoterapéutico para el diagnóstico y tratamiento de los problemas relacionados con medicamentos. Pero los criterios para clasificar un PRM en categorías, no están claros y al ser un método preestablecido de clasificación se dejan por fuera estrategias cognitivo-conductuales del usuario que pueden desembocar en algún tipo de problema o incluso otros no incluidos en el sistema de clasificación. Una debilidad del Método Dáder es la utilización del mismo cuestionario para el diagnóstico y la clasificación del problema relacionado con medicamento, y posteriormente para la evaluación del éxito de la intervención. Aunado a esto con el Método Dáder se hace una asociación causal entre paciente-medicamento-seguimiento farmacoterapéutico; pero no se brinda un análisis detallado de los mecanismos relacionados con actitudes, normas y estrategias conductuales de la persona usuaria de medicamentos.

El Método Dáder es una forma pasiva de diagnóstico para los problemas relacionados con medicamentos, es decir, la perspectiva del usuario se ve restringida al sólo hacérsele preguntas ya establecidas sobre su rutina y los efectos del medicamento. No se toman en cuenta otras posibilidades que el usuario pueda estar experimentando, tales como idiosincrasia, cultura, hábitos, creencias acerca del medicamento, características del entorno, capacidad económica, apoyo social, etc.

Con respecto a los estudios sobre variables psicosociales asociadas al consumo, adherencia y tratamiento farmacológico se hallaron interacciones entre las variables que vale la pena sintetizar. Por ejemplo, Jiménez (2004) encontró que la edad, nivel educativo, sexo y conocimiento sobre el medicamento, influyen en la decisión de cumplir con el tratamiento y en la decisión de guardar medicamentos. Rodríguez et al. (1996) encontraron asociaciones entre la posibilidad económica del paciente, capacidad para recordar indicaciones sobre el tratamiento y el apoyo familiar en adultos mayores en la adherencia al tratamiento farmacoterapéutico.

Por lo tanto, a pesar de considerar una gran cantidad de variables involucradas en el uso de medicamentos, no hay ningún estudio que determine los efectos combinados de las variables. Más investigación es necesaria no solo para determinar tales efectos combinados, sino para responder a preguntas más simples, como por ejemplo ¿Por qué consumen o dejan de consumir las personas los medicamentos? ¿Cuáles actitudes tienen un peso significativo en la decisión final de consumir o no consumir un medicamento, de guardar, regalar o recomendar el medicamento, consumir un medicamento y no otro?

\section{Referencias bibliográficas}

American Society of Hospital Pharmacists. (1993). ASHP statement on pharmaceutical care. Am J Hosp Pharm, 50, 1720-1723.

Arias, T. (1999). Glosario de Medicamentos. Washington, D.C.: Organización Panaméricana de la Salud.

Caja Costarricense de Seguro Social. (2012, Octubre-Noviembre). Medicamentos. Bienestar, 43, 1-98.

Capdevilla, C. (1981). Medicamento. En Salvarat (Ed.), Diccionario terminológico de ciencias médicas (Vol. 11). Barcelona.

Carazo, G. y Muñoz, J. (2004). Seguimiento farmacoterapéutico en Farmacias de Coтиnidad y Hogares de Ancianos de Escazú y Guadalupe. Tesis Licenciatura, Universidad de Costa Rica, San José, Costa Rica.

Federación Internacional Farmacéutica. (1993). El papel del farmacéutico en el sistema de atención de salud. Tokio, Japón: Organización Mundial de la Salud.

Grupo de investigación en Atención Farmacéutica de la Universidad de Granada. (2007). Tercer Consenso de Granada sobre Problemas Relacionados con Medicamentos (PRM) y Resultados Negativos asociados a la Medicación (RNM). Ars Pharm, 48(1), 5-17. 
Güell, A. (2002). La adherencia al tratamiento Antirretroviral en personas VIH / SIDA. Un abordaje desde la perspectiva del Trabajo Social. Revista de Trabajo Social de la CCSS, 64, 7-23.

Hepler, C.D. y Strand, L.M. (1990). Opportunities and responsibilities in pharmaceutical care. Am J Hosp Pharm, 47(3), 533-543.

Hernández, K. y Sáenz, I. (2006). Primera Encuesta Nacional de Salud (ENSA 2006): Informe técnico y primeros resultados [Versión electrónica] desde http://ccp.ucr.ac.cr/farmacoeconomia/documentos/ENSA/informetecnicoyprimerosresultadosENSA.pdf.

Jiménez-Herrera, L.G. (2008). Consumo de medicamentos alopáticos en Costa Rica. Revista Costarrisence de Salud Pública 17(32), 35-44.

Jiménez-Herrera, L.G. y Fernández-Rojas, X. (2008). Caracterización del uso de medicamentos en personas adultas mayores, Costa Rica 2007. Revista Costarrisence de Salud Pública, 17(33), 47-55.

Jiménez, E.G. y Fernández, X. (2008). Caracterización del uso de medicamentos en personas adultas mayores, Costa Rica 2007. Revista Costarricense de Salud Pública, 17(33), 47-55.

Jiménez, L.G. (2004). Análisis de la subutilización, desecho y disposición final de los medicamentos dispensados en las farmacias de la Caja Costarricense de Seguro Social, por parte de la comunidad del área de salud de Coronado. Tesis Magister Scientiae, Universidad de Costa Rica, San José, Costa Rica.

Jiménez, L.G. (2006). Consumo de medicamentos alopáticos en Costa Rica. Revista Costarricense de Salud Pública, 17(32), 1409-1429.

López, K. (2012). Consumo de medicamentos. En IAFA (Ed.), Encuesta Nacional sobre consumo de drogas. San José, Costa Rica.

Machuca-González, M., Faus-Dáder, M.J. y Fernández-Llimós, F. (2003). Método DÁDER: guía de seguimiento farmacoterapéutico: Universidad de Granada.

Mesén Arce, M. y Chamizo García, H.A. (2005). Los determinantes del ambiente familiar y la adherencia al tratamiento de la tuberculosis. Estudio de un caso urbano, el cantón de Desamparados, Costa Rica. Revistas de Ciencias Administrativas y Financieras de la Seguridad Social, 13(1), 71-82.

Ministerio de Salud. (1973). Ley 5395: Ley General de Salud. San José, Costa Rica: Asamblea Legislativa de Costa Rica.

Organización Mundial de la Salud. (1993). El papel del farmacéutico en el sistema de atención de salud. Artículo Presentado en Informe de Tokio. Retrieved. from http:// www.ops.org.bo/textocompleto/ime 9848 . pdf.

Organización Panamericana de la Salud. (2011). Guía Servicios Farmacéuticos en la Atención Primaria de Salud. Washington: Organización Mundial de la Salud.

Quesada, K. y Jaén, J. (2004). Seguimiento farmacoteratéutico con la metodología Dáder en pacientes con Diabetes Mellitus que acuden a dos farmacias de comunidad. Tesis Grado de Licenciatura y título profesional de Doctor Universidad de Costa Rica, San José, Costa Rica.

Rodríguez, V., Martínez, G. y Villalobos, H. (1996). Algunos factores psicosociales que inciden en el uso de medicamentos en el Adulto Mayor. Tesis Doctor en Medicina Familiar y Comunitaria, Universidad de Costa Rica, San José, Costa Rica.

Tinoco-Mora, Z. (2007). Selección de medicamentos. Fármacos, 18(1-2), 29-43.

Troncoso Pantoja, C., Delgado Segura, D. y Rubilar Villalobos, C. (2013). Adherencia al tratamiento en pacientes con Diabetes tipo 2. Revista Costarricense de Salud Pública, 22(1), 09-13.

Villanueva, A. (2000). Diccionario Mosby. En L. Anderson (Ed.), Diccionario Mosby (Vol. 28). Barcelona, España: Editorial Océano.

Zamora, D.M. (2005). Diccionario Médico Zamora. En Z. Editores (Ed.), Diccionario Médico Zamora. Barcelona. 
